\title{
Ochenta años atrás: esbozos de la pediatría y la cirugía pediátrica en el país
}

\author{
SERGIO ZÚÑIGA R. ${ }^{1}$ \\ 1. Profesor Asociado. Cirugía pediátrica. Facultad de Medicina, Pontificia Universidad Católica de Chile. \\ Secretario de la Sociedad chilena de Historia de la Medicina.
}

"Por un reglamento no se puede sacrificar a un enfermo; por la comodidad de un funcionario, no se le puede dejar sufrir durante horas y horas; por aceptar la tiranía de una costumbre no se puede echar a perder un Servicio que beneficia a todos".

\section{Dr. Juan Gandulfo Guerra (1895-1931)}

Según el relato de Memoria Chilena:

"Santiago, año 1932. El impacto de la crisis mundial se dejó sentir con fuerza en el país entre 1931 y 1932, estimándose por un informe de la Liga de las Naciones (World Economic Survey) que nuestra nación fue la más desvastada por la Gran Depresión. Las exportaciones de salitre y cobre se derrumbaron, provocando graves consecuencias sobre la economía interna al caer los ingresos fiscales $y$ disminuir las reservas. A mediados de 1931 la situación económica del país pareció tocar fondo, obligando a la suspensión del pago de su deuda externa por primera vez en la historia: un 16 de julio de 1931.

La crisis financiera aumentó las protestas en contra el gobierno de Carlos Ibáñez del Campo, quien se vio obligado a renunciar y partir al exilio el 26 de julio de 1931. La caída de Ibáñez dio paso a una grave crisis política sucediéndose en un poco más de un año, varios regímenes de gobierno, entre ellos la mítica República Socialista, que sólo duró doce dias. Finalmente el retorno a la normalidad política y reactivación económica comenzó con la llegada al poder de Arturo Alessandri Palma, en octubre de 1932.

La sociedad chilena se vio fuertemente sacudida por el impacto de la crisis. Miles de cesantes recorrieron las calles de ciudades y los campos; cientos de obreros salitreros volvieron sin esperanzas y recursos desde el norte. En Santiago, el gobierno a través de los Comités de Ayuda a los Cesantes debió albergar y alimentar a miles de familias; las ollas comunes proliferaron en los barrios, y mucha gente terminó viviendo en cuevas en los cerros aledaños a la ciudad".

Este es el ambiente y el momento histórico en que se publica el trabajo del Dr. Roberto Kohan sobre apendicitis aguda en el niño, en el volumen 3, año 1932, en la Revista Chilena de Pediatría y que se transcribe y comenta en el presente número. Es, también una muestra del sólido crisol en que se gestó el ejercicio de la Pediatría nacional. La realidad de la niñez chilena al comenzar el 1900 era sobrecogedora. Sólo algunos datos ilustrativos: "durante 1906 y 1907 la mortalidad de niños menores de un año fue de 41341 y 37666 respectivamente, en total 78 707", cifras que la Revista Médica califica de "asombrosas y de vergüenza nacional"". "A la peste bubónica se agregaban la epidemia de sarampión y la viruela. En Santiago el número de variolosos excedía los mil casos y en los barrios populares la gente es-

Recibido el 27 de agosto de 2012, aceptado para publicación el 21 de septiembre de 2012.

Correspondencia a:

Dr. Sergio Zúñiga Rocha

E.mail: zuniga@med.puc.cl 
condía a los variolosos y resistían los intentos de vacunación y el traslado de los enfermos al lazareto".

La inauguración oficial del Instituto de Puericultura que dirigía el Dr. Alcibíades Vicencio dio ocasión a que éste mencionara (una vez más) la aterradora mortalidad infantil nacional: en 1909 había en el país 106546 menores de un año de los que murieron 40707 , el servicio que ahora comenzaba su trabajo cuidaba al niño durante el primer año de vida y lo controlaba en ese período cada 10 a 15 días $^{3}$.

En enero de 1901 y luego de muchas gestiones de la Junta de Beneficencia y posterior a una gravísima epidemia de coqueluche y sarampión en Santiago, inicia sus actividades el Hospital de niños de la calle Matucana, que pasará a llamarse Hospital Dr. Roberto del Río en 1917, posterior al fallecimiento del distinguido pediatra. Por su parte, en 1913, comienza también sus actividades el Hospital Manuel Arriarán, en la calle Santa Rosa. Así, a comienzos de los años veinte existen en Santiago dos hospitales pediátricos y ambos dos, con sendos servicios de cirugía y médicos entrenados en el cuidado quirúrgico de niños. Los doctores Eugenio Díaz Lira y Arnulfo Johow Schaffer en el Hospital Dr. Roberto del Río y Agustín Inostroza Pérez y Juan Gandulfo Guerra en el Hospital Manuel Arriarán ${ }^{4}$. Ellos deben haber sido sin lugar a dudas, los cirujanos protagonistas de los 330 casos de la compilación del Dr. Kohan ${ }^{10}$. De este selecto grupo de eminentes cirujanos, el Dr. Juan Gandulfo Guerra (18951931) es, tal vez, uno de los más interesantes de evocar. Dotado de marcadas inquietudes sociales que volcó en actividades gremiales, lecciones para obreros y atención de policlínicos gratuitos desde su época de estudiante. Según Ignacio González, "un símbolo para los estudiantes, un héroe para los obreros y un agitador y anarquista peligroso para el gobierno"s. Excelente dibujante y de gran habilidad manual, talló en madera los grabados para la portada y todas las ilustraciones de la primera edición de Crepusculario de Pablo Neruda. Ese libro se imprime con esta dedicatoria: "A Juan Gandulfo este libro de otro tiempo"6. Escritor y poeta: ¡Siembra, Juventud! La tierra es propicia, el momento es único... Cirujano y ortopedista. Fallece trágicamente en un accidente automovilístico, a los 36 años de edad. El epígrafe de este editorial es de su autoría y revela el compromiso que caracterizaba su quehacer profesional.

En relación al tema de la apendicitis y la trayectoria de su tratamiento, este constituye una verdadera epopeya en la historia de la salud siendo el segmento etario de la niñez uno de los más afectados, ya que la enfermedad se presenta con mayor frecuencia desde la etapa pre-escolar en adelante hasta la segunda o tercera década de la vida.

La apendicitis es la inflamación del apéndice, ubicado en el ciego, que es la porción donde comienza el intestino grueso. Normalmente los casos de apendicitis aguda requieren de un procedimiento quirúrgico denominado apendicectomía, ya sea por laparotomía o laparoscopia y que no es más que la extirpación del apéndice inflamado. Este criterio quirúrgico hoy día no se discute, ya que en los casos con tratamiento tardío, la morbilidad es eleva$\mathrm{da}$, principalmente debido a complicaciones como la peritonitis y el shock séptico. Más aún, cuando el apéndice inflamado se rompe y provoca peritonitis asociada a shock séptico, todavía hoy existe riesgo de mortalidad.

A pesar de que el apéndice aparece ya representado en los dibujos anatómicos de Leonardo da Vinci en1492, es Berengario da Carpi quien lo describe por primera vez en detalle, en 1521. El órgano con forma de gusano es denominado apéndice vermiforme en 1530, por Vido Vidius (Guido Guidi). El tardío reconocimiento de la estructura por la comunidad científica tal vez, fue debido, probablemente, al hecho de que los estudios anatómicos clásicos fueron realizados en especies animales que no poseen el órgano ${ }^{7}$. Pasó mucho tiempo, y muchas personas y niños perecieron a causa del problema. Cólico Miserere, por el salmo penitencial 50, que se canta en los funerales y pasión pélvica fueron nombres populares dados a la letal enfermedad.

La primera extirpación quirúrgica apendicular publicada fue efectuada en diciembre de 1735 por Claudio Amyand, quien operó a un niño de 11 años por una hernia inguino-escrotal, encontrando al disecar el saco herniario, un 
apéndice perforado en su interior. Actualmente se denomina hernia de Amyand a aquella que contiene en su interior un apéndice atascado ${ }^{8}$.

Pero no fue sino hasta fines del siglo XIX en que se reconoció, que la mayor parte de las enfermedades inflamatorias del cuadrante inferior derecho abdominal se originaban en el apéndice y esto demostrado fundamentalmente en los trabajos de Reginald Fitz, patólogo y de los cirujanos Joseph Benjamin Murphy y Charles McBurney, todos norteamericanos. Es por eso que se señala que la afección es la quintaesencia de la cirugía estadounidense, ya que fueron ellos, los cirujanos norteamericanos, quienes describieron claramente los signos clínicos de la enfermedad y, lo más importante, plantearon la extirpación quirúrgica precoz del apéndice como el procedimiento terapéutico más recomendable ${ }^{9}$, algunos años antes del fin del siglo. Desde entonces la apendicitis es reconocida como una de las causas más frecuentes de dolor abdominal agudo o repentino en el mundo. Aproximadamente 7\% de la población será operado de una apendicectomía por causa de una apendicitis aguda.

A fines del siglo XIX, la medicina chilena estaba relativamente al día en el manejo de la enfermedad, a pesar de la distancia y la precariedad de las comunicaciones. El primer reporte sobre una operación apendicular en el país, aparece publicado en la Revista Médica de Chile, en octubre de 1894 y corresponde a un niño de 11 años operado en Valparaíso por el Dr. Olof Page. Aunque el caso se manejó a la usanza europea, es decir se esperó a que se constituyera un absceso, el que se drenó durante la operación. El niño evolucionó bien, recuperándose posteriormente ${ }^{9}$. De la misma manera fue atendido el rey de Inglaterra, Eduardo VII, en 1902, quien presentó una apendicitis días antes de su ascensión al trono.

En 1896, el Dr. Ventura Carvallo E. comunica en el Boletín de la Facultad de Medicina y Farmacia los primeros 3 casos de apendicectomía en el país, recomendando la resección del apéndice "como el único tratamiento verdaderamente curativo". Esto es reafirmado por el mismo Carvallo en el Primer Congreso Médico Latino Americano, realizado en Santiago en 1901, "Cirugía de la apendicitis. Trabajo ba- sado en 51 operaciones por apendicitis en los últimos cinco años". Esta línea de conducta fue ampliamente aceptada por los cirujanos en nuestro país. También por aquellos cirujanos que operaban niños, tanto así que en los casos que compiló el Dr. Kohan, entre 1925 y 1931 para su memoria de título, se muestra una tasa de mortalidad de $1,65 \%$ para las apendicitis, de $12 \%$ para las peritonitis apendiculares localizadas y de un $57,5 \%$ para las peritonitis generalizadas ${ }^{10}$, cifras, que en el tiempo aparecen como admirables considerando que aún no se vislumbraban sulfas ni antibióticos y la imagenología y el laboratorio clínico eran incipientes. Ese es el mérito de los primeros cirujanos de niños del país y el mérito del Dr. Kohan, de registrarlo.

Han transcurrido los años y no en vano. El desarrollo experimentado por la pediatría y la cirugía pediátrica hasta hoy habría sido impensable para nuestros antecesores de 1930. En fortuna, hemos tenido la posibilidad de participar de ese desarrollo.

\section{Referencias}

1.- http://www.memoriachilena.cl/temas/index.asp?id_ut= elimpactodelagrandpresioenchile(1929-1932)

2.- Mortalidad de niños. Rev Med Chile 1909; $37: 1$.

3.- Larraín C: La Sociedad Médica de Santiago y el Desarrollo Histórico de la Medicina en Chile. Sociedad Médica de Santiago 2002. Pág. 105.

4.- Artigas N, Montenegro E: Origen y desarrollo de los Hospitales para Niños en Santiago. RIL editores, Santiago, 2001.

5.- González I: El Juan Gandulfo de mis recuerdos. Anales Chilenos de Historia de la Medicina 1962; 2: 145-57.

6.- Neghme A: La obra literaria de los médicos chilenos. Ed. Andrés Bello. Santiago, 1984. Págs. 109-10.

7.- Ansaloni L, Catena F, Pinna AD: What Is the Function of the Human Vermiform Appendix ? Evolution-Based Surgery: A New Perspective in the Darwinian Year 2009. Eur Surg Res 2009; 43: 67-71.

8.- Hiatt JR, Hiatt N: Amyand's hernia. New England J Med 1988; 318: 1402.

9.- Reccius A: Historia y desarrollo de la Cirugía Abdominal en Chile. Ed. Zig-Zag. Santiago, 1948.

10.- Kohan R: La apendicitis Aguda en el Niño Rev Chil Pediat 1932; 3: 66-79 y 99-111. 